\title{
Anlage und Aufbau
}

Das Corpus archäologischer Quellen zur Frühgeschichte auf dem Gebiet der DDR (7. bis 12. Jahrhundert) ist nach einer einheitlichen Konzeption ausgearbeitet. Es umfaßt alle archäologischen Quellen vom Staatsgebiet der DDR, die in den angegebenen Zeitraum datiert werden können bzw. ihm nach dem heutigen Forschungsstand wahrscheinlich zugehören. Verlorengegangene oder nicht mehr nachweisbare Funde werden bearbeitet, soweit verläßliche Unterlagen zur Verfügung stehen; hierbei entstehende Unklarheiten oder Zweifel zu Einzelaussagen werden im Text deutlich herausgehoben. Dagegen finden falsch datierte oder aus anderen Gründen in der Literatur fälschlicherweise dem frühen Mittelalter zugeordnete Funde keine Berücksichtigung. Für die Vollständigkeit der Materialaufnahme sowie der Auswertung und Aufbereitung aller vorhandenen Unterlagen wird die Verantwortung von den wissenschaftlichen Bearbeitern getragen. Diese sind am Ende jeder Einzeldokumentation durch ihren Namen ausgewiesen.

Das Corpus der archäologischen Quellen zur Frühgeschichte besteht aus Text- und Abbildungsteil. Dem Ziel des Werkes entsprechend, werden grundsätzlich alle vorhandenen Funde abgebildet.

Von diesem Grundsatz wird nur in folgenden Fällen abgewichen:

Große monographisch publizierte Grabungen der letzten zwei Jahrzehnte werden im Corpus referiert. Die leicht zugänglichen Gesamtveröffentlichungen werden für jede Lieferung gesondert in einer Liste ausgewiesen (vgl. S. 401).

Große Grabungen, deren wissenschaftliche Bearbeitung nicht abgeschlossen ist, werden gemäß dem erreichten Bearbeitungsstand resümierend dargestellt. Die Publikation eines repräsentativen Querschnittes des Fundmaterials wird in diesen Fällen angestrebt.

Gegenstände aus Metall, Knochen oder Bein und anderen Materialien werden in jedem Falle abgebildet, auch wenn von einem Fundplatz mehrere Stücke des gleichen Typs vorliegen. Bei Keramikkomplexen erfolgt dagegen eine repräsentative Auswahl aus der Gesamtzahl der Gefäßoberteile. Wandungs- und Bodenteile werden nur ausnahmsweise abgebildet.

Alle Abbildungen sind als Strichzeichnungen angelegt. Die einheitlichen und nicht gesondert nachgewiesenen Maßstäbe betragen für Keramik 1:4, für alle übrigen Funde 1:2. Für wenige Sonderfälle, bei denen von dieser Regel abgewichen werden muß - etwa Schwerter (Maßstab 1:4) oder Hacksilberschmuck (Maßstab 1:1) -, erfolgt ein gesonderter Nachweis des Maßstabes am unteren Rand der Tafel.

Pläne oder Planskizzen von Burgwällen, Grabungspläne und ähnliche Unterlagen erscheinen als Textabbildungen im Zusammenhang mit der zugehörigen Fundstelle. Dieser Abbildungsteil beruht auf der Basis des gegenwärtigen Arbeitsstandes.

Die Gliederung des Corpus der archäologischen Quellen zur Frühgeschichte auf dem Gebiet der DDR folgt der politischen Einteilung der DDR in Bezirke und Kreise. Dabei wurde angestrebt, geschlossene Gebiete möglichst zusammenhängend zu erfassen. 
Das Werk wird in fünf Lieferungen eingeteilt, die jeweils folgende Bezirke umfassen:

1. Lieferung Bezirk Rostock (Westhälfte), Bezirk Schwerin, Bezirk Magdeburg

2. Lieferung Bezirk Rostock (Osthälfte), Bezirk Neubrandenburg

3. Lieferung Bezirk Frankfurt/Oder, Bezirk Potsdam, Berlin

4. Lieferung Bezirk Cottbus, Bezirk Dresden, Bezirk Karl-Marx-Stadt, Bezirk Leipzig

5. Lieferung Bezirk Halle, Bezirk Gera, Bezirk Erfurt, Bezirk Suhl

Sämtliche Kreise wurden mit fortlaufenden Kennummern versehen. Diese Numerierung richtet sich nach der obengenannten Anordnung der Bezirke und ist innerhalb eines Bezirkes so vorgenommen worden, daß benachbarte Kreise aufeinander folgen. Auf eine alphabetische Anordnung der Kreise wurde also verzichtet; die Orientierung wird dem Benutzer durch das beigegebene Kreisverzeichnis erleichtert.

Innerhalb eines Kreises sind die Fundorte alphabetisch geordnet und die Fundstellen fortlaufend numeriert. Die sich aus der Nummer des Kreises und der der Fundstelle zusammensetzende Kennummer wird einem jeden Fundort vorangestellt.

Beispiel: 1/1 Darsow

Als Fundort wird jeweils die Gemarkung oder der Ortsteil aufgeführt. Wenn Gemarkung und politische Gemeinde nicht identisch sind, wird die Angabe der Gemeinde dem Namen der Gemarkung nachgeordnet. In gleicher Weise wird bei den Ortsteilen verfahren.

Beispiel: 1/2 Feldhusen, Gem. Pötenitz 4/21 Malpendorf, Ot. von Neubuckow

Sind in der Literatur unzutreffende Angaben des Fundortes eingeführt worden, ist unter dem fälschlicherweise angeführten Fundort ein Verweis auf den richtigen Gemarkungsnamen angebracht. Funde aus ehemaligen Staatsforsten sind unter dem Namen der Gemarkung zu finden, der das Fundstellengebiet inzwischen zugeordnet wurde.

Diese territorialen Angaben stützen sich auf das „Verzeichnis der Gemeinden und Ortsteile der Deutschen Demokratischen Republik“, Berlin 1966 (Redaktionsschluß vom 31. Juli 1965). Das gilt auch für die Einteilung und Zuordnung der Kreise, mit Ausnahme des Kreises 19, Osterburg-Seehausen. Nach dem 31. Juli 1965 geschaffene Stadtkreise, Veränderungen in der Zuordnung von Gemeinden oder Gemarkungen bzw. Ortsteilen u. a. finden im Corpus keine Berücksichtigung.

Die zum Text gehörigen Abbildungen sind über die Kennzahl der jeweiligen Fundstelle aufzufinden, die im Tafelteil vor der ersten Abbildung eines Fundkomplexes eingedruckt ist. Der Erschließung des Werkes dienen Abbildungsunterschriften. Diese enthalten die Kennzahlen der jeweils behandelten Fundkomplexe sowie die Fundorte, Kreis und Bezirk. Die Beschreibung eines jeden Fundplatzes erfolgt nach einheitlichem Schema, gegliedert nach folgenden Punkten:

Mbl. (Meßtischblatt): Angabe der Nummer des Meßtischblattes und der Hoch- und Rechtswerte, sofern die Fundstelle genau lokalisiert ist.

Fst. (Fundstelle): Kurzgefaßte Beschreibung der Lage des Fundplatzes.

Beispiele: Nr. 1; 1,2 sö; auf flachem Horst im Niederungsgelände. $\ldots ; 0,5 \mathrm{w} ;$ Sportplatz s Dorfstraße.

Die erstgenannte Zahl gibt die amtliche Fundstellennummer im Archiv der zuständigen Forschungsstelle für Ur- und Frühgeschichte an. Ist eine solche amtliche Numerierung der Fundstelle bisher nicht erfolgt, erscheint ...;. Der antlichen Nummer der Fundstelle folgt die Angabe über ihre Lage zum heutigen Ort. Die Entfernung in Kilometern und die Himmelsrichtung [n, s, ö, w] sind - sofern ergänzende Hinweise fehlen (z. B. 2,0 sw vom Ortsrand) - stets auf die Mitte des Ortes (in der 
Regel Kirche oder Dorfanger) bezogen. Zusätzliche Angaben umfassen die allgemeine Lagebeschreibung, Hinweise auf Flurnamen und eine kurze Charakterisierung der Geländeverhältnisse.

FA (Fundart): Die kurzgefaßte Fundartbestimmung umfaßt in der Regel die Begriffe Burgwall, Siedlung, Gräberfeld, Einzelfund und dient der leichteren Nutzung des Corpus.

FU (Fundumstände): Angabe des Fundjahres und kurze Beschreibung der Umstände, unter denen der Fund geborgen wurde. Soweit es sich nicht um eine moderne Ausgrabung handelt, bzw. bei kleineren Rettungsgrabungen werden zugleich die wesentlichen Befunde mitgeteilt.

Grbg. (Grabung): Kurze Zusammenfassung der wesentlichen Ergebnisse archäologischer Ausgrabungen. Bei Gräberfeldern können die das einzelne Grab betreffenden Befunde unter der folgenden Position „Funde“ abgehandelt werden, um eine doppelte Darstellung zu vermeiden.

Funde: Stichwortartige Fundbeschreibung. Liegen von einem Fundplatz mehrere geschlossene Fundkomplexe vor, werden sie getrennt beschrieben; die Reihenfolge ergibt sich aus den Angaben zu FU bzw. Grbg. Innerhalb eines Komplexes wird in der Regel folgende Ordnung angewandt: Münzen, Schmuck aus Edelmetall und anderen Metallen, Waffen und zugehörige Ausrüstungen, Werkzeuge und Geräte aus Metall und aus anderen Materialien, Keramik, Tierknochen und übrige Funde (z. B. Eisenschlacke, Webgewichte). Funde, die vom gleichen Fundplatz stammen, jedoch zu anderen Perioden gehören, werden am Ende der Beschreibung erwähnt.

$\mathrm{Zu}$ jedem einzelnen Fund (bei Keramik gewöhnlich für den ganzen Komplex) werden Sammlung, Inventarnummer und vorliegende Veröffentlichungen nachgewiesen (in Klammern gesetzt und der Fundbeschreibung nachgestellt). Die Abbildungsnummern beziehen sich auf die einzelnen Abbildungen. Die Auffindung eines Fundkomplexes im Abbildungsteil erfolgt über die Kennummer des Fundplatzes.

Dat. (Datierung): Die Angaben zur Datierung werden, ausgehend vom gegenwärtigen Forschungsstand, von jedem Autor gegeben. Ist die Datierung durch den Zusatz vermutl. oder wahrscheinl. eingeschränkt worden, handelt es sich um Fundplätze, von denen nur wenige Oberflächenfunde vorliegen und deren Zeitdauer daher unsicher bleibt.

Erw. (Erwähnung): Auf Urkunden oder chronikalischen Nachrichten beruhende Angaben, die sich entweder direkt oder infolge des archäologisch nachgewiesenen Besiedlungszeitraumes mit hoher Wahrscheinlichkeit auf den Fundplatz beziehen.

Dtg. (Deutung): Kurze, vom Autor ausgearbeitete Deutung der Befunde. Weicht sie von bereits publizierten Aussagen ab, wird darauf gesondert hingewiesen. Die Verwendung der einschränkenden Begriffe vermutl. und wahrscheinl. erfolgt dann, wenn der Fundplatz noch nicht ausreichend erforscht ist.

Nachw (Nachweis): Nachweis der Museen, Sammlungen und Institutionen, in denen das archäologische Fundgut und die Archivmaterialien aufbewahrt werden. Diese Einrichtungen werden alphabetisch gezählt. Durch beigeordnete Buchstaben wird im Text der Dokumentation jeweils auf sie verwiesen.

Die offizielle Bezeichnung der Museen und Sammlungen ist aus dem beigefügten Verzeichnis zu ersehen (vgl. S. XVII).

Lit. (Literatur): Literaturangaben werden in einem solchen Umfang vorgenommen, daß alle Veröffentlichungen mit wesentlichen Aussagen über den Fundplatz erfaßt sind. Deshalb werden die Erstveröffentlichungen stets und spätere insoweit zitiert, als sie neue, über die erstgenannte hinausgehende Angaben enthalten. Monographien und größere Aufsätze werden bibliographisch vollständig zitiert, bei kurzen Fundberichten und Mitteilungen erfolgt dagegen lediglich die Angabe von Zeitschriften- 
titel, Jahrgang und Seitenzahl. Häufig wiederkehrende Titel lassen sich über das Literaturverzeichnis, gekürzte Zeitschriftentitel über das Sigelverzeichnis erschließen. Auf Veröffentlichungen wird im Text durch in Klammern gesetzte arabische Zahlen verwiesen.

Bem. (Bemerkungen): In einigen Fällen sind Angaben mitzuteilen, die sich nicht in das der Dokumentation zugrundeliegende Schema einfügen lassen. 


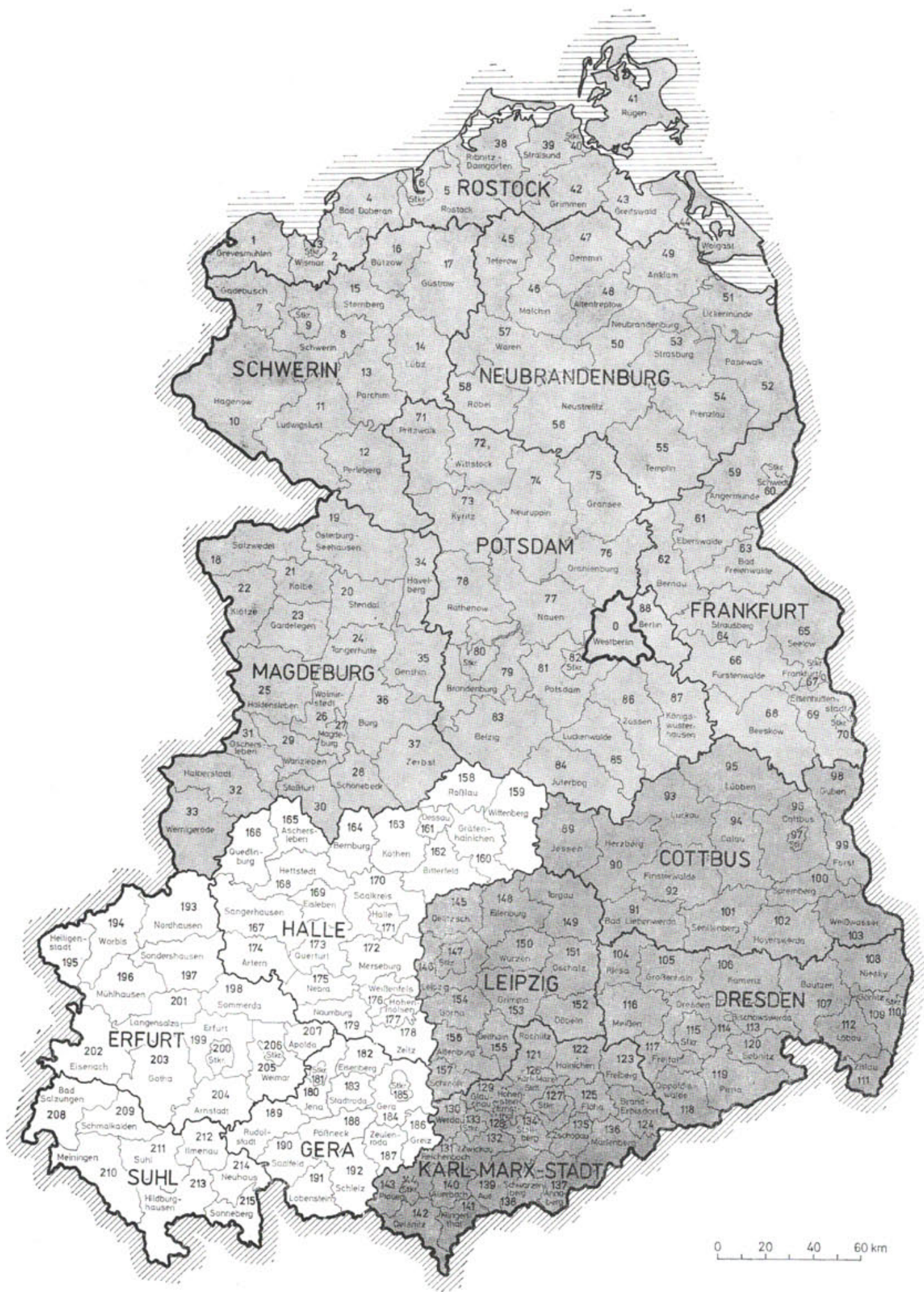

Karte 1. Deutsche Demokratische Republik. Übersicht über die Kreise mit Kennzahlen. 